\title{
Enteropatía congénita y trasplante intestinal
}

\author{
Olivier Goulet
}

Centro de Referencia de Enfermedades Digestivas Raras; Departamento de Gastroenterología-Hepatología

Pediátrica y Nutrición, Hôpital Necker-Enfants Malades, París, Francia

\section{Palabras clave}

Insuficiencia intestinal · Citrulina Enteropatía

congenital · Nutrición parenteral $\cdot$ Insuficiencia

hepática - Trasplante intestinal · Trasplante hepático •

Nutrición parenteral domiciliaria

\section{Extracto}

La insuficiencia intestinal (II) exige el uso de nutrición parenteral (NP). Entre las causas de II prolongada grave destacan el síndrome del intestino corto, trastornos de la motilidad grave (aganglionosis total o subtotal o síndrome de seudoobstrucción intestinal crónica) y enfermedades congénitas del desarrollo de los enterocitos. Puede aparecer insuficiencia hepática grave en pacientes con II como consecuencia de una enfermedad digestiva subyacente y una NP no adaptada. La sepsis relacionada con el catéter y/o la trombosis vascular extensa pueden impedir el mantenimiento de una NP segura y eficiente. Por tanto, el tratamiento de pacientes con II exige la identificación precoz del proceso y el análisis de su riego de irreversibilidad. La cronología de la derivación para trasplante intestinal continúa siendo una cuestión crucial. En consecuencia, la asistencia debe incluir tratamientos adaptados a cada una de las etapas de la II, basándose en la puesta en práctica de un enfoque mul- tidisciplinario en centros que cuenten con gastroenterología pediátrica, experiencia en NP, programa de NP domiciliaria, cirugía pediátrica y programa de trasplantes de hígado e intestino.

Copyright (C) 2006 Nestec Ltd., Vevey/S. Karger AG, Basel

La insuficiencia intestinal (II) es un proceso de malabsorción intestinal grave que exige la instauración de nutrición parenteral (NP). Las enteropatías congénitas que dañan la mucosa intestinal llevan a la II irreversible. Dado que el riesgo de complicaciones de la NP permanente es elevado, se plantea la posible necesidad de un trasplante intestinal (TxI).

\section{Definición de insuficiencia intestinal}

'Insuficiencia intestinal' se define como la reducción crítica de la masa intestinal funcional, por debajo de la cantidad mínima necesaria para una digestión y absorción adecuadas, a fin de satisfacer las necesidades de nutrientes y líquidos corporales para el mantenimiento de la salud en adultos o el crecimiento en niños. No obstante, esta definición no está admitida uniformemente. Algunos dan a entender que la II se define mejor en términos

\section{KARGER}

Fax +4161306 1234 E-Mail karger@karger.ch www.karger.com
C 2006 Nestec Ltd. Vevey/S. Karger AG, Basel $0252-8185 / 06 / 0641-0025 \$ 23.50 / 0$

Accesible online en: www.karger.com/ans
Olivier Goulet, Reference Center for Rare Digestive Diseases

Department of Pediatric Gastroenterology-Hepatology and Nutrition Hôpital Necker-Enfants Malades, 149, rue de Sèvres FR-75743 Paris Cedex 15 (France)

Tel. +331444944 11, Fax +331444925 01, E-Mail olivier.goulet@nck.ap-hop-paris.fr 
de pérdidas de energía fecal que de longitud del intestino residual en pacientes con síndrome del intestino corto (SIC) [1]. Otro enfoque consiste en evaluar el grado de II con arreglo a la cantidad de NP exigida para el mantenimiento en adultos y el crecimiento en niños. Además de los criterios anatómicos y nutricionales para definir la II, recientemente se ha propuesto un marcador biológico de la masa intestinal funcional [2]. La citrulina es un aminoácido no esencial producido predominantemente por los enterocitos [3]. Los niveles plasmáticos de citrulina pueden medirse fácilmente y reflejan la pérdida de masa funcional en pacientes con SIC y en pacientes con diversos grados de atrofia de las vellosidades o que experimentan un rechazo de injertos intestinales con atrofia de las vellosidades [4]. En un estudio reciente, realizado en pacientes pediátricos con SIC tratados con hormona del crecimiento humana recombinante, los niveles plasmáticos de citrulina se redujeron significativamente tras el tratamiento [5]. El criterio actual para definir la II sigue siendo la incapacidad para mantener el crecimiento en niños sin dependencia permanente de la NP.

\section{Causas de la insuficiencia intestinal}

La evolución natural de la II en niños es variable y está influida en gran medida por el trastorno subyacente responsable del menoscabo de la función intestinal. El tipo más corriente de II proviene de la infección gastrointestinal. No obstante, se prevee que los niños afectos de este tipo de II aguda se recuperen por completo con un tratamiento apropiado. No obstante, la diarrea prolongada postenteritis sigue siendo una causa principal de II en países en vías de desarrollo [6]. En países desarrollados, la II está dominada por el inicio congénito o precoz de la enfermedad grave y permanente que requiere NP prolongada o indefinida. En el pasado, el SIC fue una de las primeras causas identificadas de II prolongada [7]. No obstante, con el uso creciente y satisfactorio de NP a largo plazo durante las tres últimas décadas, han aparecido otras varias causas de II, por ejemplo, graves y extensos trastornos de la motilidad, entre los que destacan aganglionosis subtotal, síndrome de seudo-obstrucción intestinal crónica y enfermedades congénitas del desarrollo de los enterocitos [8].

\section{Enteropatía congénita causante de insuficiencia intestinal}

Se propuso un intento de clasificar la diarrea intratable de la lactancia (DIL) según el grado de atrofia de las vellosidades, basándose en criterios inmunohistológicos que resaltan el papel de las células $\mathrm{T}$ activadas en la mucosa intestinal [9]. En un examen multicéntrico del ESPGHAN se recogieron casos de DIL y atrofia de las vellosidades con características definidas con precisión por microscopía óptica, lo que permitió la categorización de diversos tipos de DIL [10]. El primero se caracterizaba por una infiltración de células mononucleares de la lamina propria y se consideraba asociado a células $T$ activadas. La segunda configuración histológica comprendía un inicio precoz de diarrea grave con atrofia de las vellosidades, sin infiltración de células mononucleares de la lamina propria, y anomalías histológicas específicas que afectaban al epitelio. Hasta la fecha se han identificado varios tipos de anomalías epiteliales primarias inductoras de DIL. La primera descrita fue la atrofia de las microvellosidades (AMV) o enfermedad de inclusión de microvellosidades y, más recientemente, la enteropatía en penacho o displasia epitelial intestinal (DEI) [11]. Algunos pacientes pequeños para la edad gestacional presentan anomalías fenotípicas que corresponden a la DEI [12].

\section{Atrofia de las microvellosidades}

En 1978, Davidson et al. [13] informaron de 5 lactantes con diarrea grave, persistente, que se inició durante el periodo neonatal y en quienes la microscopía óptica reveló la presencia de una atrofia hipoplásica de las vellosidades de las criptas. El examen con microscopio electrónico de biopsias de intestino delgado en 3 de estos pacientes mostró intensas anomalías en el ribete en cepillo y un aumento de cuerpos de tipo liposómico; en otro de estos pacientes se observaron quistes intracitoplasmáticos con arquitectura de ribete en cepillo. También se describieron niños con inclusiones citoplasmáticas características de la membrana del ribete en cepillo [14]. A partir de estas descripciones clínicas e histológicas se determinó que la AMV era una entidad patológica separada dentro del síndrome de la DIL. En su forma típica, la AMV es un trastorno congénito de las células epiteliales intestinales, que se presenta inicialmente como diarrea neonatal intratable [14].

\section{Expresión clínica}

En general, los lactantes presentan una diarrea acuosa grave dentro de los primeros días después del nacimien- 
to. Los volúmenes de las deposiciones pueden llegar hasta $250-300 \mathrm{ml} / \mathrm{kg}$ de peso corporal/día y las concentraciones fecales de electrolitos son similares a las del líquido del intestino delgado. Este trastorno conlleva especialmente un riesgo vital, dado que la diarrea masiva da lugar a deshidratación rápida, desequilibrios electrolíticos y la subsiguiente descompensación metabólica en el curso de unas pocas horas. La diarrea acuosa grave persiste a pesar del reposo intestinal. Entre los diagnósticos diferenciales cabe destacar la diarrea congénita por cloruros y la diarrea por malabsorción de sodio, que puede distinguirse fácilmente de la AMV por medio de la determinación de los electrolitos circulantes y fecales [15, 16]. Por el contrario, la presentación clínica de la AMV también puede ser muy atípica, con un intestino delgado lleno y distendido y un colon que simula un síndrome seudooclusivo. El inicio más corriente de la diarrea de la AMV tiene lugar en el curso de los primerísimos días (forma de inicio precoz o congénita). No obstante, en unos pocos pacientes, el inicio de la diarrea puede retrasarse (primer mes) y ser menos grave (forma de inicio tardío).

\section{Análisis histopatológicos}

El diagnóstico puede establecerse fácilmente a partir de un examen con microscopio óptico de muestras de biopsia duodenal o yeyunal. En la tinción con hematoxilina y eosina, la mucosa aparece aplanada con atrofia hipoplásica de las vellosidades. Muy característico de este trastorno es la acumulación de gránulos secretores positivos al ácido peryódico de Schiff (APS) dentro del citoplasma apical de los enterocitos [17, 18] (fig. 1). A nivel de la ultraestructura se observan microvellosidades escasas o ausentes e inclusiones citoplasmáticas de microvellosidades de enterocitos. Estos cambios anatómicos definen esta entidad [19]. Recientemente se ha propuesto un nuevo y valioso instrumento para el diagnóstico de la AMV por microscopía óptica [20, 21]. La CD-10 es una peptidasa neutra asociada a la membrana que, según se ha demostrado, posee una configuración de tinción lineal del ribete en cepillo en el intestino delgado normal. En contraste con esta tinción superficial en diferentes condiciones (intestino normal, celiaquía, enteropatía autoinmune y alergia), todos los casos de AMV muestran una inmunorreactividad intracitoplasmática de CD-10 en los enterocitos superficiales [20]. Se obtuvieron resultados similares con APS, antígeno carcinoembrionario policlonal y fosfatasa alcalina, tres colorantes conocidos por revelar tinción citoplasmática de los enterocitos superficiales en la AMV [22]. Al microscopio electrónico, las células epiteliales superficiales muestran microvellosidades ausentes o macroscópicamente anormales y numerosos cuerpos vesiculares de diversos tamaños, así como inclusiones de microvellosidades características (fig. 2). Las células de las criptas son casi normales en términos morfológicos. No contienen mayores números de vesículas apicales y cuerpos vesiculares. En el epitelio del intestino grueso también se observan inclusiones de microvellosidades y un aumento de gránulos secretores. Esto reviste especial importancia, dado que este tejido es más fácilmente accesible para la biopsia, especialmente durante la primera lactancia.

Fisiopatología y modo de transmisión

En la AMV también se discutió como mecanismo etiopatógeno una anomalía en el tránsito de enterocitos inmaduros y/o en fases de diferenciación a traves de la membrana intestinal $[19,22]$. Una consecuencia funcional directa de esta anomalía de la membrana es la II completa. Se ha especulado que esta enfermedad se asocia a un trastorno del citoesqueleto del enterocito. El citoesqueleto produce un ensamblaje anormal de microvellosidades. Se describió que la distrofia de las microvellosidades intestinales era una hipotética variante de la AMV [22]. No obstante, la patogénesis subyacente de la AMV sigue sin conocerse en todos sus extremos. Cuando se analiza el recambio de sacarasa-isomaltasa, como proteína representativa del ribete en cepillo, resulta evidente que las vías constitutivas, directas e indirectas, permanecen intactas en la AMV [19]. Dado que tanto en la AMV congénita como en la de inicio tardío las microvellosidades se presentan casi normales en regiones en las que se acumulan primero los gránulos secretores, no es probable que una anomalía de la biosíntesis de las microvellosidades sea la base de la enfermedad. Recientemente, investigando los gránulos secretores, Phillips et al. [23] dieron a entender que la AMV implica una anomalía de la exocitosis del glucocáliz que podría deteriorar las funciones normales de las células.

Considerando el número de casos con hermanos afectados y la frecuencia de consanguinidad entre los pacientes, esta enfermedad parece transmitida como rasgo autosómico recesivo [24]. Aunque hasta la fecha no se ha identificado ningún gen candidato, se ha informado de la presencia de AMV en una muchacha con hipocondroplasia autosómica dominante [25]. La anomalía génica de esta hipocondroplasia ha sido localizada recientemente en la región cromosómica 4p16.3. Esto podría contribuir a aclarar la base genética de las AMV. 
Fig. 1. Tinción con APS. A Mucosa normal, APS normal, tinción del ribete en cepillo. B Acumulación anormal de APS, material positivo en el citoplasma apical de células epiteliales en la enfermedad de inclusión de microvellosidades.
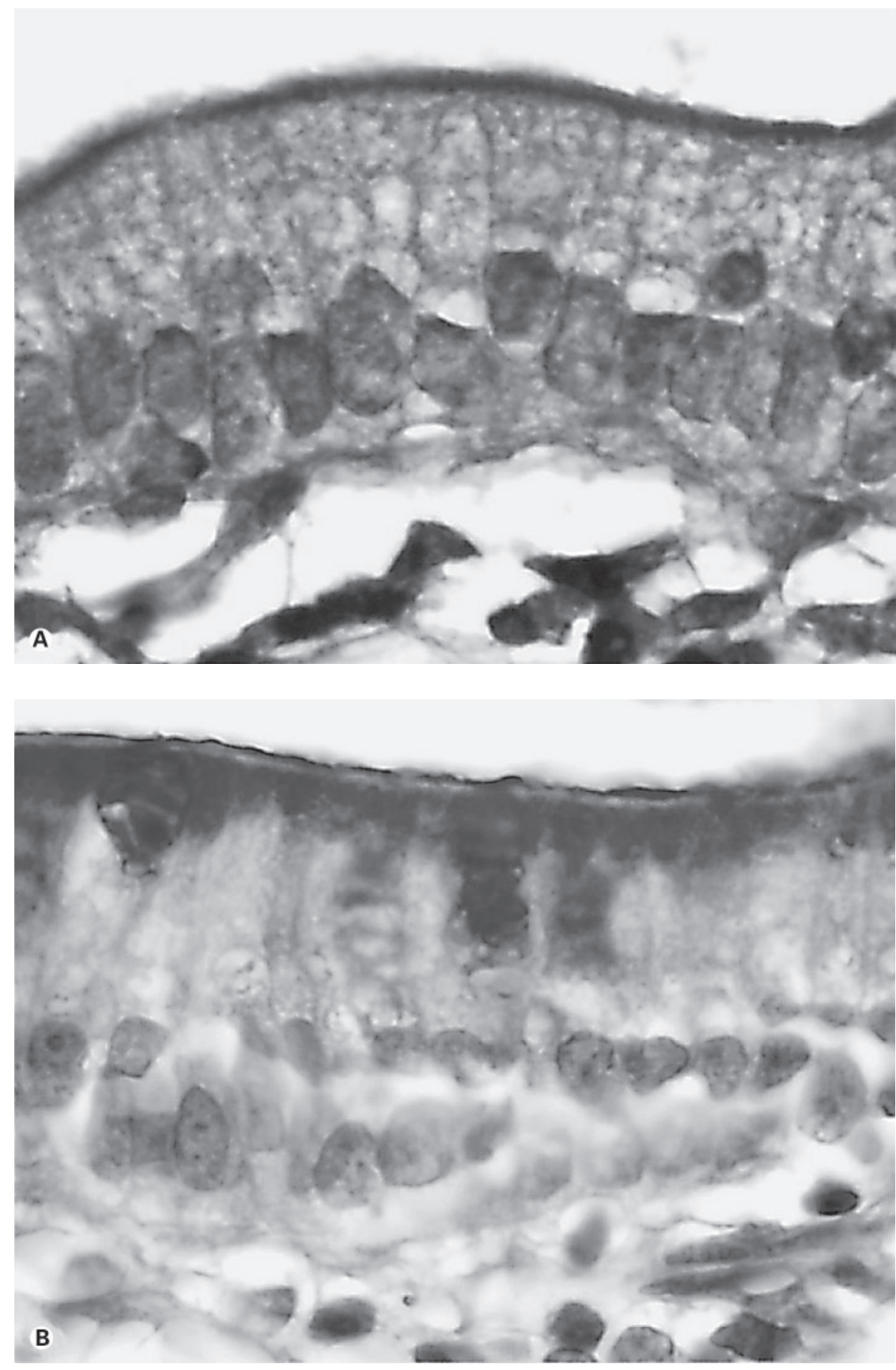

Resultados a largo plazo

El examen multicéntrico más extenso de 23 pacientes con AMV [14] reveló una esperanza de vida extremadamente precaria, con una tasa de supervivencia en un año inferior al 25\%. La mayoría de los niños falleció por complicaciones sépticas, insuficiencia hepática o descompen- sación metabólica. Son pocos los casos de AMV, especialmente la forma de inicio tardío y las formas con emisión de deposiciones limitadas, que pueden sobrevivir con NP sólo parcial. El tratamiento con corticosteroides, calostro o factor de crecimiento epidérmico no ha sido satisfactorio; sin embargo, se ha utilizado octreótido con éxito par- 
Fig. 2. Enfermedad de inclusión de microvellosidades. Microscopía electrónica de una muestra de biopsia yeyunal. El ribete en cepillo está casi ausente. El citoplasma contiene una inclusión de microvellosidades.

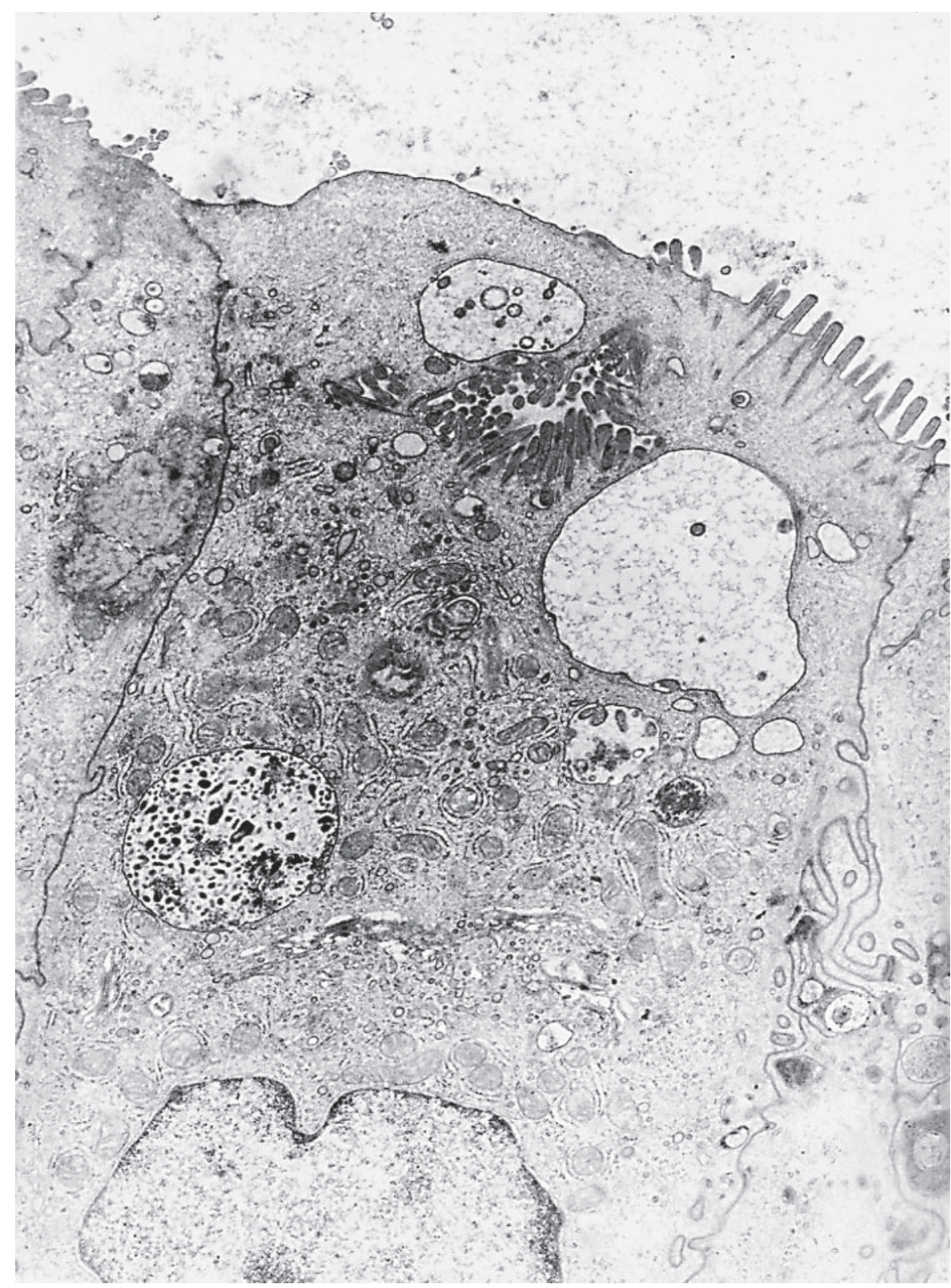

cial en un paciente [14]. Contrariamente a los resultados iniciales obtenidos antes de la década de los 80, la NP permite actualmente la supervivencia de la mayoría de los lactantes y los niños. No obstante, las complicaciones asociadas a una NP inadecuada limitan la supervivencia a largo plazo. Además, en ausencia de signos de insuficiencia renal asociada, algunos de estos lactantes y niños sufren desequilibrios hidroelectrolíticos crónicos y acidosis con deterioro subsiguiente del crecimiento. Otros, debido a episodios repetidos de deshidratación asociados a ingestiones inapropiadas de fósforo y calcio, presentan nefrocalcinosis. Por último, incluso con una NP a largo plazo adecuada y un crecimiento normal, persisten emisiones considerables y molestas de deposiciones que, a menudo, exigen sustituciones diarias de líquidos y electrolitos con el riego elevado de deshidratación grave. Por lo tanto, el TxI puede ser el único tratamiento definitivo de esta rara enfermedad intestinal.

Se han descrito varios casos de trasplantes satisfactorios en la AMV [26-29]. El trasplante puede ser de intestino aislado [26] o combinado con hígado [27, 28]. Sigue debatiéndose la cuestión de si el colon debe ser trasplan- 
tado o no junto con el intestino delgado. Recientemente, informamos de pacientes con AMV congénita de inicio precoz [29]. Dos pacientes fallecieron antes de que se pudieran incluir en una lista de espera para trasplante de intestino delgado y un paciente sigue a la espera de dicho trasplante. Hemos practicado TxI cadavérico en 7 pacientes de edades comprendidas entre 3 y 11 años utilizando tacrolimus, esteroides y bloqueantes de la IL-2. Se realizaron tres TxI y 4 asociados a hígado. El trasplante de colon derecho se efectuó en 5 casos ( 2 TxI aislados). Sobrevivieron 6 pacientes $(86 \%)$ con una mediana de seguimiento de 3 años (intervalo: 1-8 años). Se logró la supresión completa de la NP en todos los pacientes. Por vez primera, el TxI solo o en combinación con hígado ofrecía a niños con AMV una perspectiva a largo plazo [29, 30].

\section{Displasia epitelial intestinal o enteropatía en penacho}

Reifen et al. [31] describieron 3 casos de diarrea neonatal grave con cuadros epiteliales anormales bajo la denominación de 'enteropatía en penacho'. Identificamos a 9 casos de diarrea neonatal grave que diferían claramente de la AMV [11]. Estudios ulteriores en estos pacientes demostraron que la DEI es un trastorno epitelial constitutivo en el que participa tanto el intestino delgado como el colon [32]. En nuestra experiencia, la DEI parece frecuente en pacientes de origen árabe y de Oriente Medio y el Norte de África. Una característica principal de esta enfermedad es su heterogeneidad clínica e histológica y su asociación a malformaciones u otras enfermedades epiteliales.

\section{Expresión clínica}

Normalmente, los pacientes presentan diarrea grave durante las primeras semanas de vida. La mayoría tiene padres consanguíneos y/o un hermano afectado. Algunos miembros de la familia afectados fallecieron durante los primeros meses de vida con diarrea grave de origen desconocido. En la mayor parte del tiempo, la diarrea persistía a pesar del reposo intestinal; sin embargo, la mortalidad era inferior a la observada en la AMV. Los intentos de utilizar nutrición enteral continua con un hidrolizado de proteínas o aminoácidos empeoraban la diarrea. Los recién nacidos presentan desnutrición rápidamente, así como una malnutrición de proteína y energía grave.

\section{Presentación histológica}

La atrofia de las vellosidades está presente pero su gravedad es variable. En su forma característica, las ano- malías se localizan principalmente en el epitelio y comprenden una desorganización de los enterocitos superficiales con agolpamiento focal similar a penachos (fig. 3). Estos 'penachos' característicos de epitelio sobresaliente, descritos por vez primera por Reifen et al. [31], se observan hacia el extremo de la vellosidad y pueden afectar hasta al 70\% de éstas. El proceso de formación del penacho no se limita al intestino delgado sino que afecta también a la mucosa colónica [11]. Este cuadro puede observarse también en el epitelio de las criptas y, además, las criptas presentan a menudo un aspecto anormal, con dilatación que hace recordar a seudoquistes, y regeneración anormal con ramificación [11]. El estudio de los componentes de la membrana basal demostró la deposición anormal de los proteoglicanos laminina y sulfato de heparano en este nivel, en comparación con muestras de biopsia de pacientes con celiaquía o enteropatía autoinmune [11]. En relación con los controles se registró una deposición débil e irregular de laminina en la superficie de contacto epitelial de la lamina propria, mientras que los depósitos del proteoglicano sulfato de heparano eran grandes y laminares, indicativos del desarrollo anormal de la membrana basal. Éstos pueden ser el origen de las anomalías epiteliales. Por otra parte, observamos un incremento de la expresión inmunohistoquímica de desmogleína en la DEI y cambios en la ultraestructura de los desmosomas, que aumentaron tanto en longitud como en número [32] (fig. 4).

Se sospecha de DEI más frecuentemente después de excluir la AMV en cortes teñidos con APS. Su diagnóstico final se establece después de efectuar biopsias intestinales repetidas, que cambian desde subnormales al comienzo de la vida (sólo atrofia de las vellosidades inespecífica con o sin infiltración de células monocelulares de la lamina propria) hasta los penachos característicos. Otra dificultad está relacionada con la infiltración de la lamina propria por células T. Los infiltrados de células $T$ refuerzan el diagnóstico de enteropatía de origen inmunitario, tal como se ha descrito previamente en especial en ausencia de penachos. Cabe especular que la adhesión celular defectuosa incrementa la permeabilidad intestinal con la reacción inflamatoria subsiguiente. Murch et al. [33] describieron este tipo de lesión en lactantes con displasia epitelial.

\section{Trastornos Asociados}

Se comunicaron varios casos de DEI asociados a anomalías fenotípicas específicas, por ejemplo, el síndrome de Dubowitz o síndrome de malformación [34, 35]. Recientemente, se informó en 4 niños de una asociación 
Fig. 3. Displasia epitelial intestinal. Atrofia parcial de las vellosidades con hiperplasia de las criptas y/o aspecto de criptas seudoquísticas, cuadros de ramificación y desorganización del epitelio superficial.

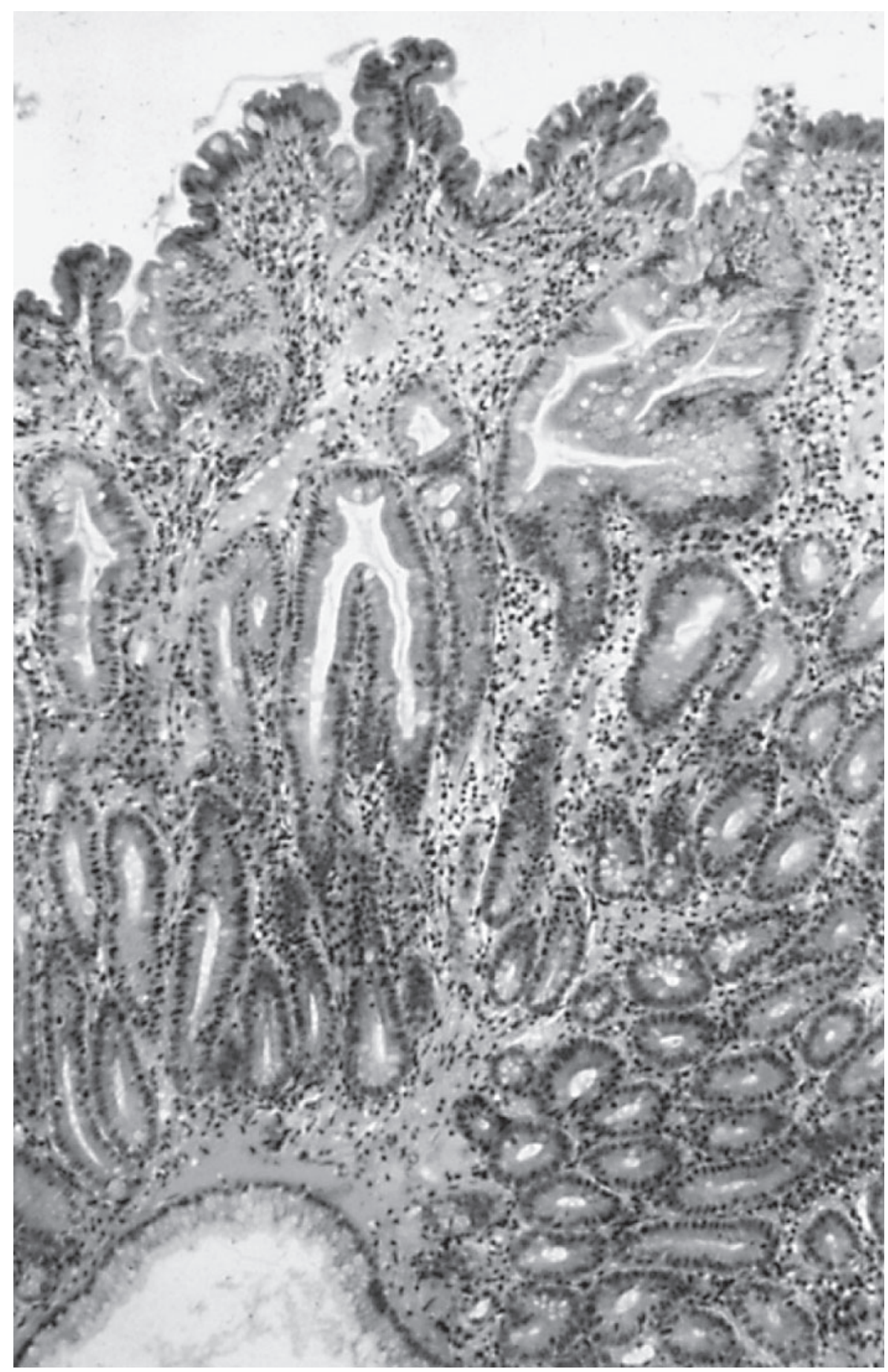

entre DIL congénita y atresia coanal [34]. Hemos observado malformaciones, incluyendo atresia rectal o esofágica, y recientemente informamos de una queratitis puntiforme inespecífica que afectaba aproximadamente al $60 \%$ de los pacientes [36]. Esta queratitis es muy curiosa, dado que se trata también de una enfermedad epitelial que podría contribuir a aclarar los mecanismos moleculares de la enfermedad epitelial intestinal. El hecho de que algunos niños carezcan de síntomas oftalmológicos ilustra la probable heterogeneidad de la enfermedad.

Destacamos por su interés que Lachaux et al. [37] describieron recientemente el caso de un recién nacido con 


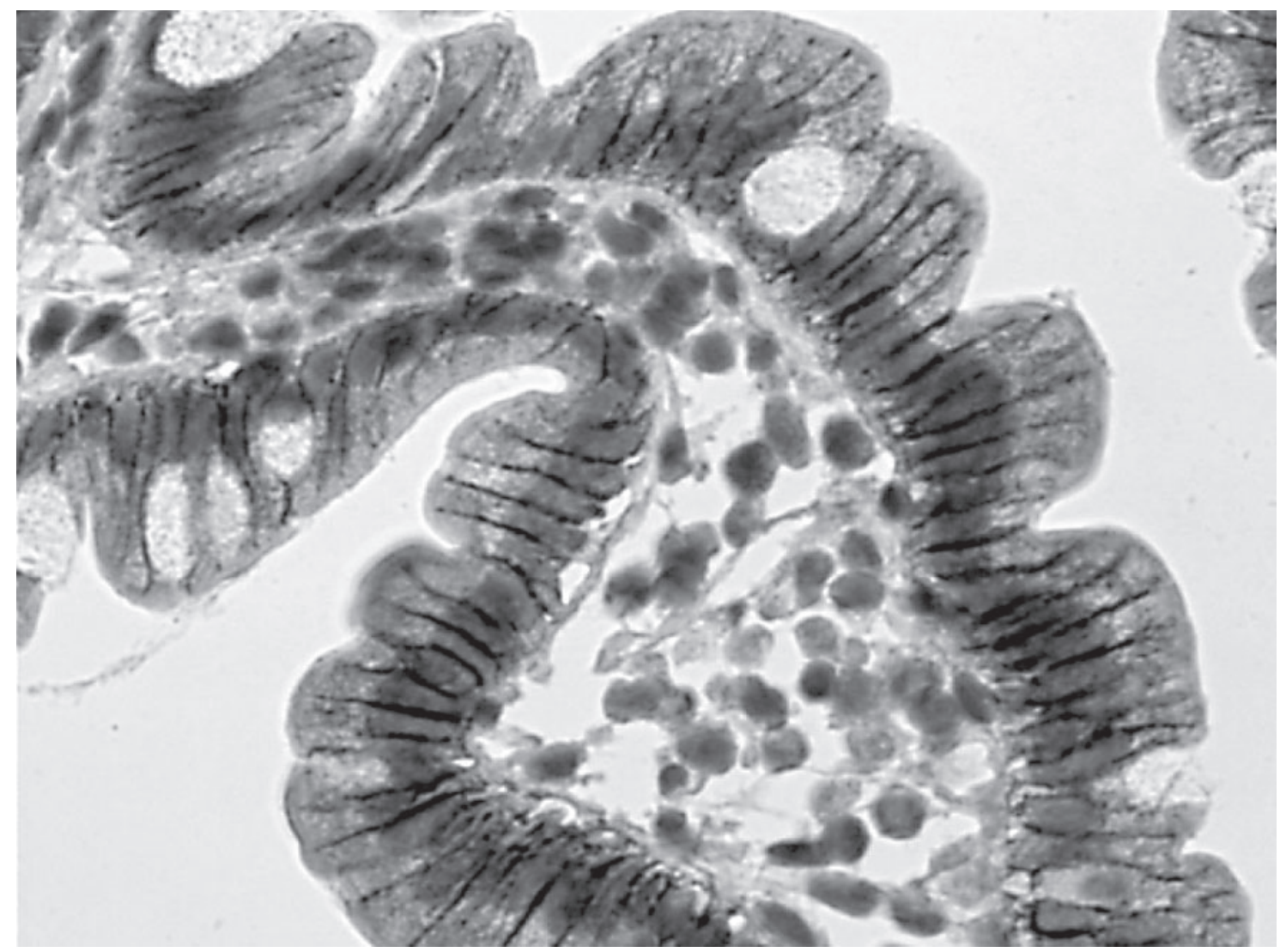

Fig. 4. DEI. Aumento de la expresión de la tinción de desmogleina de la unión estrecha en un paciente con DEI.

atresia de píloro y diarrea intratable. El examen con microscopía óptica reveló una descamación extensa desde el fundus hasta el recto, con permanencia de sólo unas pocas células epiteliales en las bases de las criptas. La microscopía electrónica del epitelio intestinal reveló desmosomas normales y, sin embargo, una segmentación localizada entre la lamina propria y el polo basal de los enterocitos. Esta descripción inicial guardaba relación con una deficiencia congénita de la integrina $\alpha_{6} \beta_{4}$, conocida por ser defectuosa en la epidermolisis bullosa.

Fisiopatología y modo de transmisión

Se demostró que la DEI se asociaba a una membrana basal anormal en comparación con las de muestras de biopsia de pacientes con celiaquía o enteropatía autoinmune [11]. Las moléculas de la membrana basal participan en interacciones de células mesenquimatosas epiteliales, que son instrumentales en el desarrollo y la diferenciación intestinales [38]. Se observaron alteraciones indicativas de interacciones normales entre células y en- tre célula y matriz en pacientes con DEI, sin signos de anomalías en la polarización y la proliferación de células epiteliales [32]. Las alteraciones comprendían una distribución anormal de moléculas de adhesión, la integrina $\alpha_{2} \beta_{1}$ a lo largo del eje cripta-vellosidad. La integrina $\alpha_{2} \beta_{1}$ participa en la interacción de células epiteliales con diversos componentes de la membrana basal, como la laminina y el colágeno. Los mecanismos fisiopatológicos que resultan en un aumento de la expresión inmunohistoquímica de desmogleina y los cambios ultraestructurales en los desmosomas siguen sin conocerse a ciencia cierta [32]. Un ratón con menoscabo del cambio funcional en el gen que codifica el factor de transcripción Elf3 presenta características morfológicas similares a la displasia epitelial en lactantes [39]. En este modelo de ratón existe una morfogénesis anormal de las vellosidades, pero las células progenitoras de las criptas son normales. Los enterocitos en ratones con deficiencia de Elf3 producen niveles bajos del receptor de tipo 2 del factor de crecimiento transformador $\beta$, que induce la diferenciación de los 
epitelios intestinales inmaduros. Actualmente, en lactantes humanos, sigue pendiente de determinación la naturaleza primaria o secundaria de las modificaciones observadas. La presencia de penachos puede proporcionar pistas importantes. Los penachos corresponden a células epiteliales no apoptóticas en los extremos de las vellosidades, que ya no están en contacto con la membrana basal. En términos especulativos, la responsabilidad de este efecto puede recaer en una anomalía de la apoptosis normal de los enterocitos al final de su ciclo vital o en un contacto intercelular alterado. La naturaleza primaria o secundaria de la formación de penachos sigue pendiente de determinación.

Hasta la fecha se sospecha del origen genético de este trastorno a partir de la asociación evidente de consanguinidad entre los padres y/o hermanos afectados. Estas características permiten suponer una transmisión autosómica recesiva. El gen implicado en esta enfermedad congénita, hereditaria, autosómica recesiva, todavía no ha sido identificado. Esta enteropatía parece más frecuente que la AMV, especialmente en la población de Oriente Medio.

\section{Resultados}

La diarrea neonatal que resiste a todos los tratamientos requiere NP permanente. No obstante, parece que algunos lactantes desarrollan un fenotipo más leve que otros [40]. Debido a la función intestinal residual parcial y a la cantidad limitada de emisión de deposiciones, algunos pacientes necesitan únicamente NP a largo plazo parcial con infusiones 3 a 4 veces por semana. No obstante, debe efectuarse una vigilancia minuciosa para evitar un retraso de crecimiento progresivo. En la mayoría de los pacientes, la gravedad de la malabsorción intestinal y la diarrea los hacen completamente dependientes de la NP diaria, a largo plazo, con el riesgo subsiguiente de complicaciones. La DEI puede ser otra indicación para el TxI [41, 42].

\section{Otras enteropatías congénitas}

La llamada 'diarrea fenotípica', que es un síndrome de DIL asociado a anomalías fenotípicas e inmunodeficiencia, es también una enfermedad rara, descrita recientemente [12]. Los pacientes afectados presentan diarrea en el curso de los 6 primeros meses de vida $(<1$ mes en la mayoría de los casos) y comparten varias características clínicas. Son pequeños para la edad gestacional y presentan un fenotipo anormal [12]. Todos tienen dismorfismo facial con frente prominente, nariz ancha e hipertelorismo. Presentan una anomalía del cabello bien diferencia- da, la tricorrexis nudosa, en la cual el cabello está ensortijado, es difícil de manejar, puede arrancarse fácilmente y está mal pigmentado. Estas características se observan también en niños que provienen de Oriente Medio. Entre las formas congénitas de displasia capilar, la tricorrexis nudosa es muy corriente y puede presentarse en diversos procesos patológicos [43, 44]. Estos pacientes presentan también respuestas de anticuerpos defectuosas a pesar de niveles séricos normales de inmunoglobulinas, y pruebas cutáneas defectuosas, específicas de antígeno, a pesar de respuestas proliferativas positivas in vitro [12]. Las muestras de la biopsia de intestino delgado en pacientes con diarrea sindromática muestran atrofia de las vellosidades moderada o grave, con infiltrados inconstantes de células mononucleares de la lamina propria y ausencia de anomalías epiteliales. Histológicamente, no se detectan anomalías específicas. El pronóstico de este tipo de DIL es reservado, dado que la mayoría de los pacientes muere entre los 2-5 años de edad. Algunos sufren insuficiencia hepática de inicio precoz [12]. La causa de esta diarrea es desconocida y la relación entre bajo peso al nacer, dismorfismo, diarrea grave, tricorrexis e inmunodeficiencia no se conoce a ciencia cierta.

\section{Tratamiento de la insuficiencia intestinal}

\section{Nutrición parenteral a largo plazo}

Sea en el hospital o a domicilio, la NP a largo plazo expone al paciente a complicaciones graves, entre las que destacan sepsis y trombosis relacionadas con el catéter, insuficiencia hepática y osteopatía [45]. El cuidado diario del catéter y la prevención de complicaciones sépticas son esenciales [46]. Deben realizarse pruebas de función hepática con regularidad, debido al riesgo de colestasis y lesión hepática. La ecografía destinada a indagar la presencia de barro o colelitiasis debe repetirse por lo menos dos veces al año en pacientes que conservan la vesícula biliar. Además, conviene examinar la mineralización ósea mediante DEXA y evaluar el estado de fósforo y calcio; la dieta debe modificarse para prevenir la aparición de osteopatías relacionadas con la NP.

\section{Factores y tratamiento de la insuficiencia hepática}

La insuficiencia hepática es la complicación más frecuente y grave en pacientes con II. La expresión 'insuficiencia hepática relacionada con NP' debe ser sustituida por 'insuficiencia hepática relacionada con II'. De hecho, los factores más importantes que inducen colestasis e insuficiencia hepática terminal guardan relación con el de- 
terioro de la función intestinal y el sobrecrecimiento bacteriano. La insuficiencia hepática se agrava adicionalmente por una NP inadecuada.

A continuación se enumeran los factores principales relacionados con la insuficiencia hepática:

(1) Interrupción del ciclo enterohepático (enfermedad o resección ileal) [47, 48]

(2) Estasis intestinal con sobrecrecimiento bacteriano intraluminal subsiguiente y/o translocación (endotoxinemia) [49] 51]

(3) Sepsis recurrente en relación con el catéter [50,

(4) Prematurez [51]

(5) Ingestión inadecuada de macronutrientes, como infusión de NP continua con ingestión excesiva de glucosa, conducente a hiperinsulinismo y esteatosis [52], y provisión inadecuada de aminoácidos [49]

(6) Ingestión excesiva de ácidos grasos poliinsaturados de cadena larga con su lipoperoxidación asociada [53, 54], y contenido elevado en fitoesteroles de emulsiones basadas en aceite de soja [55]

(7) Sobrecarga de aluminio, hierro y/o cromo [56-58].

Los lactantes prematuros y/o pequeños para la edad gestacional con enterocolitis necrosante grave presentan un riego especialmente elevado de tener insuficiencia hepática y fallo hepático terminal prematuro, debido a la combinación de prematuridad, suboclusión, sepsis gramnegativa, reposo intestinal prolongado, infusión de NP continua en lugar de cíclica y sepsis recurrente relacionada con el catéter.

La prevención o resolución de la insuficiencia hepática puede ser posible por medio de:

(1) Estimulación del eje enterobiliar, asegurando la ingestión de triglicéridos de cadena larga o leche materna, o por inyección de análogos de colecistoquinina [59]

(2) Supresión del sobrecrecimiento bacteriano intraluminal causado por estasis intestinal mediante la administración de metronidazol [60] y/o la realización de enteroplastia ahusada

(3) Administración de ácido ursodesoxicólico (30 mg/ $\mathrm{kg} / \mathrm{día}$ ) para atenuar la insuficiencia hepática [61]

(4) Prevención de la sepsis relacionada con el catéter mediante el cuidado diario apropiado de la vía $[50,51]$

(5) Adaptación del suministro de NP por medio de:

(a) Limitación del aporte de glucosa para reducir la acumulación de grasa en el hígado [62, 63].

(b) Uso del tipo y cantidad apropiados de emulsión grasa intravenosa para proporcionar ácidos grasos esenciales, reduciendo la carga de glucosa y limitando la peroxidación [53-55]. El reciente desarrollo de emulsiones grasas basadas en aceite de oliva puede reducir la peroxidación [54]. La administración diaria de lípido intravenoso no debe sobrepasar los $2-2,5 \mathrm{~g} / \mathrm{kg}$

(6) Control de la provisión y la velocidad de suministro de los lípidos y/o cese de la administración intravenosa de lípidos inmediatamente tras la aparición de trombocitopenia, hiperbilirrubinemia y/o ictericia [53]

(7) Uso de nuevas soluciones pediátricas de aminoácidos adaptados, que proporcionan aminoácidos apropiados más taurina [49]

(8) Suministro de NP cíclica, que reduce el hiperinsulinismo y la esteatosis hepática [64]

(9) Adaptación del aporte de hierro y reducción del contenido en aluminio de las soluciones de NP [65].

Con el cuidado y la prevención apropiados la insuficiencia hepática puede retrasarse o incluso evitarse, y el paciente puede ser mantenido en un estado nutricional y general que conlleve un mejor pronóstico cuando se requiera la realización de un trasplante de intestino delgado aislado en caso de II irreversible.

\section{Trasplante intestinal}

Sea cual fuere la causa de la II prolongada o irreversible, la NP a largo plazo es la piedra angular del tratamiento. La NP domiciliaria permite que lactantes y niños crezcan en el entorno psicológico más idóneo $[66,67]$. Es preciso demostrar la irreversibilidad de la II antes de considerar la realización de un TxI.

Cuando el TxI llega a ser la opción terapéutica indicada, son imperativas extensas discusiones multidisciplinarias entre cirujanos de trasplantes y gastroenterólogos pediátricos. Las evaluaciones y las decisiones se basan en la aparición de las complicaciones enumeradas durante el seminario desarrollado durante el Simposio Internacional sobre Trasplante de Intestino Delgado, celebrado en Miami, en septiembre de 2003. Estos criterios son similares a los comunicados en el artículo de opinión de la Sociedad Norteamericana de Trasplantes [68] y siguen siendo objeto de debate, especialmente los relacionados con trombosis y sepsis vasculares:

(1) Insuficiencia hepática (niveles de bilirrubina $>3$ $\mathrm{mg} / \mathrm{dl}$, esplenomegalia, trombocitopenia, varices gastroesofágicas, coagulopatía, sangrado estomal, fibrosis hepática o cirrosis)

(2) Trombosis de los accesos venosos principales $(>2$ trombosis en las venas subclavias, yugulares o femorales). En nuestro servicio se deja un acceso en el lecho de la vena cava superior 
(3) Sepsis recurrente relacionada con el catéter (2 episodios al año de sepsis sistémica secundaria a la infección de la vía, un episodio de fungemia relacionada con la vía, shock séptico o síndrome disneico agudo)

(4) Enfermedad subyacente que causa pérdidas incontrolables de agua y electrolitos y frecuente deshidratación aguda grave.

Estos criterios representan normas y no recomendaciones formales. Deben ser sopesados frente a los riesgos del TxI.

Las contraindicaciones para el TxI no difieren de las correspondientes a otros órganos sólidos. Debido a los riesgos del TxI, especialmente en casos de mal estado del receptor en el momento del trasplante y el tamaño del conjunto de donantes, el concepto de que el paciente debe tener el potencial para obtener algún beneficio obvio del procedimiento debe seguir siendo prioritario.

Entre las demás contraindicaciones destacan las discapacidades neurológicas congénitas o adquiridas, las enfermedades extradigestivas que conllevan riesgo vital, los trastornos inmunitarios congénitos o adquiridos, neoplasias malignas no operables y penetración vascular insuficiente para garantizar un acceso venoso central fácil durante un periodo de hasta 6 meses después del TxI.

\section{Tipo de trasplante}

Los pacientes con II irreversible e insuficiencia hepática terminal (cirrosis hepática) son claros candidatos de procedimientos de salvamento como el trasplante combinado de hígado e intestino delgado (TIHx). Los pacientes con fibrosis hepática grave son más difíciles de tratar. Es imprescindible la realización de biopsias hepáticas repetidas en el curso de 6 a 12 meses y evaluaciones minuciosas de la hipertensión portal. Además, es difícil evaluar la cantidad de tejido hepático funcional necesaria para contrarrestar la agresión de la desviación portal durante el procedimiento de trasplante. Los niños con fibrosis hepática grave, avanzada y progresiva, son habitualmente candidatos de TIHx. No obstante, algunos pacientes dependientes de la NP, con disfunción hepática avanzada, pueden experimentar una recuperación funcional y bioquímica del hígado que se produce paralelamente al rescate intestinal autólogo. Varios casos consecutivos de colestasis grave se recuperaron por completo tras la suspensión de la administración intravenosa de lípidos [69]. La histología no es siempre pronóstica de la recuperación funcional del hígado [70]. No obstante, tras la manifestación de cirrosis, la supervivencia en 1 año es solo del 20 al $30 \%$ [71].
Cronología de la derivación para trasplante intestinal

Los factores que influyen sobre la supervivencia de niños con II derivados para practicarles un TxI son bien conocidos; entre ellos destacan los siguientes: edad $<1$ año; enfermedad quirúrgica; fibrosis o cirrosis a la espera de trasplante; niveles de bilirrubina $>3 \mathrm{mg} / \mathrm{dl}$ y trombocitopenia [71]. Se ha confirmado que los pacientes derivados a centros de cuidados intensivos para TIHx están debilitados, presentan varias complicaciones, así como periodos de hospitalización prolongados. El informe UNOS indica que la mortalidad de pacientes en listas de espera de TxI es mayor que en otras listas de espera de trasplantes [72], lo cual puede explicar las bajas tasas de supervivencia de pacientes e injertos descritas en diversos estudios [73]. El Registro de Trasplantes Intestinales confirmó que los trasplantes realizados en pacientes que lo esperaban en su domicilio frente a los que lo esperaban en el hospital presentaban una mejor supervivencia en un año $(74 \%$ frente a $59 \%$; $p<0,00001)$ [73]. La tendencia a trasplantar proporcionalmente a más pacientes que permanecían a la espera en sus domicilios es un importante factor contributivo a la reciente mejoría de las tasas de supervivencia de injertos y pacientes [73].

Son pocos los centros que gestionan todas la etapas de la II, desde su inicio hasta el TxI incluyendo el programa de NP domiciliario. Se informó de una notable discrepancia en el estado clínico entre niños derivados para TxI de centros con y sin equipos de cuidados nutricionales [74]. El tratamiento a largo plazo de la II se ha convertido en un problema muy importante, tal como dejan entrever estudios recientemente publicados [75]. Una red nacional o internacional podría proporcionar datos sobre la II, en los que deberían basarse las necesidades de atención sanitaria [76], y posibilitar la optimación del tratamiento, la mejora del enfoque médico y la promoción de investigación básica y aplicada en este ámbito.

De hecho, el tratamiento apropiado de la II requiere centros de referencia que utilicen estrategias terapéuticas basadas en un enfoque multidisciplinario en el que participarían gastroenterólogos pediátricos, bromatólogos, cirujanos pediátricos, enfermeras especializadas, dietistas, trabajadores sociales y psicólogos. Este enfoque integrado debe adaptarse a cada tipo y etapa de II, incluyendo la NP domiciliaria y un programa de trasplantes. 


\section{Conclusión}

El tratamiento precoz de la II en centros de referencia, a cargo de un grupo multidisciplinario, es la clave para la identificación oportuna de la II y su inversión, la obtención de mejores resultados y la realización de un TxI a tiempo. Tal como ha sido recalcado recientemente por un informe del Grupo de Trabajo de Gastroenterología y Nutrición Pediátricas, es necesaria la investigación en to- dos los ámbitos de adaptación tras una resección de intestino delgado y la identificación de los genes implicados en la enteropatía congénita o el síndrome de seudoobstrucción intestinal [77]. También es imperativo evaluar los costes respectivos de la NP domiciliaria a largo plazo y el TxI y seleccionar la mejor opción para incrementar al máximo la calidad de vida de los niños afectados y sus familias.

\section{Bibliografía}

1 Nordgarrd I, Hansen BS, Mortensen PB: Importance of colonic support for energy absorption as small-bowel failure proceeds. Am J Clin Nutr 1996;64:222-231.

-2 Crenn P, Coudray-Lucas C, Thuillier F, Cynober L, Messing B: Postabsorptive plasma citrulline concentration is a marker of absorptive enterocyte mass and intestinal failure in humans. Gastroenterology 2000;119:14961505.

-3 Crenn P, Vahedi K, Lavergne-Slove A, Cynober L, Matuchansky C, Messing B: Plasma citrulline: A marker of enterocyte mass in villous atrophy-associated small bowel disease. Gastroenterology 2003;124:1210-1219.

4 Pappas PA, Saudubray JM, Tzakis AG, et al: Serum citrulline and rejection in small bowel transplantation: a preliminary report. Transplantation 2001;72:1212-1216.

5 Dabbas-Tyan M, Colomb V, Rosilio M, Landais P, Ricour C, Goulet O: Evaluation of the effect of recombinant human growth hormone (rhGH) treatment of children with short bowel syndrome. J Pediatr Gastroenterol Nutr 2000; 31:S165-S166.

66 Walker-Smith JA: Diarrhoeal disease: current concepts and future challenges. Malnutrition and infection. Trans R Soc Trop Med Hyg 1993;87(suppl 3):13-15.

7 Goulet O, Révillon Y, Jan D, et al: Neonatal short bowel syndrome. J Pediatr 1991;119:1823.

8 Goulet O, Ruemmele F, Lacaille F, Colomb V: Irreversible intestinal failure. J Pediatr Gastroenterol Nutr 2004;38:250-269.

-9 Cuenod B, Brousse N, Goulet O, et al: Classification of intractable diarrhea in infancy using clinical and immunohistological criteria. Gastroenterology 1990;99:1037-1043.

10 Goulet O, Brousse N, Canioni D, et al: Syndrome of intractable diarrhoea with persistent villous atrophy in early childhood: A clinicopathological survey of 47 cases. J Pediatr Gastroenterol Nutr 1998;26:151-161.

11 Goulet O, Kedinger M, Brousse N, et al: Intractable diarrhea of infancy: a new entity with epithelial and basement membrane abnormalities. J Pediatr 1995;127:212-219.
12 Giraut D, Goulet O, Ledeist F, et al: Intracta- 23 Phillips AD, Brown A, Hicks S, Schuller S, ble diarrhea syndrome associated with phenotypic abnormalities and immune deficiency. $\mathrm{J}$ Pediatr 1994;125:36-42.

13 Davidson GP, Cutz E, Hamilton JR, Gall DG: Familial enteropathy: a syndrome of protracted diarrhea from birth, failure to thrive, and hypoplastic villous atrophy. Gastroenterology 1978;75:783-790.

14 Phillips AD, Schmitz J: Familial microvillous atrophy: A clinicopathological survey of 23 cases. J Pediatr Gastroenterol Nutr 1992;14: 380-396.

15 Holmberg C: Congenital chloride diarrhea Clin Gastroenterol 1980;3:583-602.

16 Booth IW, Stange G, Murer H, Fenton TR, Milla PJ: Defective jejunal brush-border $\mathrm{Na}+/$ $\mathrm{H}+$ exchange: a cause of congenital secretory diarrhoea. Lancet 1985;i:1066-1069.

17 Phillips AD, Jenkins P, Raafat F, WalkerSmith JA: Congenital microvillous atrophy: specific diagnostic features. Arch Dis Child 1985;60:135-140.

18 Phillips AD, Szfranski M, Man L-Y, Wall W: Periodic acid Schiff staining abnormality in microvillous atrophy: photometric and ultrastructural studies. J Pediatr Gastroenterol Nutr 2000;30:34-42.

19 Phillips A, Fransen J, Hauri HP, Sterchi E: The constitutive exocytotic pathway in microvillous atrophy. J Pediatr Gastroenterol Nutr 1993;17:239-246.

20 Groisman GM, Amar M, Livne E: CD10: A valuable tool for the light microscopic diagnosis of microvillous inclusion disease (familial microvillous atrophy). Am J Surg Pathol 2002; 26:902-907.

21 Youssef N, Canioni D, Ruemmele F, et al: CD10 expression in microvillous inclusion disease (abstract). J Pediatr Gastroenterol Nutr 2003; 36:563.

22 Raafat F, Green NJ, Nathavitharana KA, Booth IW: Intestinal microvillous dystrophy: a variant of microvillous inclusion disease or a new entity? Hum Pathol 1994;25:1243-1248. Murch SH, Walker-Smith JA, Swallow DM: Acetylated sialic acid residues and blood group antigens localise within the epithelium in microvillous atrophy indicating internal accumulation of the glycocalyx. Gut 2004;53:17641771.

24 Nathavitharana KA, Green NJ, Raafat F, Booth IW: Siblings with microvillous inclusion disease. Arch Dis Child 1994;71:71-73.

25 Heinz-Erian P, Schmidt H, Le Merrer M, et al: Congenital microvillous atrophy in a girl with autosomal dominant hypochondroplasia. J Pediatr Gastroenterol Nutr 1999;28:203-205.

-26 Oliva MM, Perman JA, Saavedra JM, et al: Successful intestinal transplantation for microvillous inclusion disease. Gastroenterology 1994; 106:771-774.

27 Herzog D, Atkinson P, Grant D, et al: Combined bowel-liver transplantation in an infant with microvillous inclusion disease. J Pediatr Gastroenterol Nutr 1996;22:405-408.

28 Randak C, Langnas AN, Kaufman SS, et al: Pretransplant management and small bowelliver transplantation in an infant with microvillous inclusion disease. J Pediatr Gastroenterol Nutr 1998;27:333-337.

29 Ruemmele FM, Jan D, Lacaille F, et al: New perspectives for children with microvillous inclusion disease: early small bowel transplantation. Transplantation 2004;77:1024-1028.

30 Croft NM, Howatson AG, Ling SC, et al: Microvillous inclusion disease: an evolving condition. J Pediatr Gastroenterol Nutr 2000;31: 185-189.

31 Reifen RM, Cutz E, Griffiths AM, Ngan BY, Sherman PM: Tufting enteropathy: a newly recognized clinicopathological entity associated with refractory diarrhea in infants. J Pediatr Gastroenterol Nutr 1994;18:379-385.

32 Patey N, Scoazec JY, Cuenod-Jabri B, et al: Distribution of cell adhesion molecules in infants with intestinal epithelial dysplasia (tufting enteropathy). Gastroenterology 1997;113: 833-843.

3 Murch S, Graham A, Vermault A, et al: Functionally significant secondary inflammation occurs in a primary epithelial enteropathy. $\mathrm{J}$ Pediatr Gastroenterol Nutr 1997;24:467. 
-34 Krantz M, Jansson U, Rectors S, Ryd W, Kristiansson B: Hereditary intractable diarrhea with choanal atresia. A new familial syndrome. J Pediatr Gastroenterol Nutr 1997;24:470.

35 Abely M, Fromont Hankard G, et al: Intractable infant diarrhea with epithelial dysplasia associated with polymalformation. J Pediatr Gastroenterol Nutr 1998;27:348-352.

36 Djeddi D, Verkarre V, Talbotec C, et al: Tufting enteropathy and associated disorders (abstract). J Pediatr Gastroenterol Nutr 2002;34: 446.

- 37 Lachaux A, Bouvier R, Loras I, et al: Isolated deficient $\alpha 6 \beta 4$ integrin expression in the gut associated with intractable diarrhoea. J Pediatr Gastroenterol Nutr 1999;29:395-401.

- 38 Simon-Assmann P, Kedinger M: Heterotypic cellular cooperation in gut morphogenesis and differentiation. Cell Biol 1993;4:221-230.

-39 Ayn NG, Waring P, Ristevski S, et al: Inactivation of the transcription factor Elf3 in mice results in dysmorphogenesis and altered differentiation of intestinal epithelium. Gastroenterology 2002;122:1455-1466.

-40 Cameron DJS, Barnes GL: Successful pregnancy outcome in tufting enteropathy. J Pediatr Gastroenterol Nutr 2003;36:158.

-41 Lacaille F, Cuenod B, Colomb V, et al: Successful combined liver and small bowel transplantation in a child with epithelial dysplasia. J Pediatr Gastroenterol Nutr 1998;27:230-233.

-42 Paramesh AS, Fishbein T, Tschernia A, et al: Isolated small bowel transplantation for tufting enteropathy. J Pediatr Gastroenterol Nutr 2003;36:138-140.

-43 Itin PH, Pittelkow MR: Trichothiodystrophy: review of sulfur-deficient brittle hair syndromes and association with the ectodermal dysplasia. J Am Acad Dermatol 1990;22:705717.

44 Verloes A, Lombet J, Lambert Y, et al: Trichohepato-enteric syndrome: further delineation of a distinct syndrome with neonatal hemochromatosis phenotype, intractable diarrhea, and hair anomalies. Am J Med Genet 1997;68: 391-395.

45 Shulman RJ, Phillips S: Parenteral nutrition in infants and children. J Pediatr Gastroenterol Nutr 2003:36:587-607.

- 46 Colomb V, Fabeiro M, Dabbas M, Goulet O, Merckx J, Ricour C: Central venous catheterrelated infections in children on long-term home parenteral nutrition: incidence and risk factors. Clin Nutr 2000;19:355-359.

47 Colomb V, Goulet O, Rambaud C, et al: Long term parenteral nutrition in children: liver and gallbladder disease. Transplant Proc 1992;24: 1054-1055.

48 Moss RL, Das JB, Raffensperger JG: Total parenteral nutrition-associated cholestasis: clinical and histopathological correlation. J Pediatr Surg 1993;28:1270-1275.

49 Forchielli ML, Walker WA: Nutritional factors contributing to the development of cholestasis during total parenteral nutrition. Dev Pediatr 2003;50:245-267.
50 Colomb V, Jobert-Giraud A, Lacaille F, Goulet O, Fournet JC, Ricour C: Role of lipid emulsions in cholestasis associated with long-term parenteral nutrition in children. J Parenter Enteral Nutr 2000;24:345-350.

51 Moseley RH: Sepsis-associated cholestasis. Gastroenterology 1997;112:302-305.

- 52 Lienhardt A, Rakotoambinina B, Colomb V, et al: Insulin secretion and sensitivity in children on cyclic total parenteral nutrition. J Parenter Enteral Nutr 1998;22:382-386.

$\checkmark 53$ Belli DC, Fournier LA, Lepage G, et al: Total parenteral nutrition-associated cholestasis in rats: comparison of different amino acid mixtures. J Parenter Enteral Nutr 1987;11:6773.

54 Goulet $\mathrm{O}$, de Potter S, Antebi H, et al: Longterm efficacy and safety of a new olive oil-based intravenous fat emulsion in pediatric patients: a double-blind randomized study. Am J Clin Nutr 1999;70:338-345.

55 Clayton PT, Whitfield P, Iyer K: The role of phytosterols in the pathogenesis of liver complications of pediatric parenteral nutrition. Nutrition 1998;14:158-164.

-56 Popinska K, Kierkus J, Lyszkowska M, et al: Aluminum contamination of parenteral nutrition additives, amino acid solutions, and lipid emulsions. Nutrition 1999; 15:683-686.

57 Ben Hariz M, Goulet O, De Potter S, et al: Iron overload in children receiving prolonged parenteral nutrition. J Pediatr 1993;123:238241.

58 Moukarzel A, Song MK, Buchman AL, et al: Excessive chromium intake in children receiving total parenteral nutrition. Lancet 1992; 339:385-388.

59 Ling PR, Sheikh M, Boyce P, et al: Cholecystokinin (CCK) secretion in patients with severe short bowel syndrome (SSBS). Dig Dis Sci 2001;46:859-864.

60 Capron JP, Gineston JL, Herve MA: Metronidazole in prevention of cholestasis associated with total parenteral nutrition. Lancet 1983;i:446-447.

61 Heubi JE, Wiechmann DA, Creutzinger V, et al: Tauroursodeoxycholic acid (TUDCA) in the prevention of total parenteral nutrition-associated liver disease. J Pediatr 2002;141:237_ 242.

62 Bresson JL, Narcy P, Putet G, et al: Energy substrate utilization in infants receiving total parenteral nutrition with different glucose to fat ratios. Pediatr Res 1989;25:645-648.

63 Kaufman SS: Prevention of parenteral nutrition-associated liver disease in children. Pediatr Transplant 2002;6:37-42.

64 MacFie J: Cyclic parenteral nutrition. Nutrition 1997;13:46-48.
65 Advenier E, Landry C, Colomb V, et al: Aluminum contamination of parenteral nutrition and aluminum loading in children on longterm parenteral nutrition. J Pediatr Gastroenterol Nutr 2003;36:448-453.

66 Colomb V, Goulet O, Ricour C: Home enteral and parenteral nutrition. Baillieres Clin Gastroenterol 1998;122:877-894.

67 Scolapio JS: A review of the trends in the use of enteral and parenteral nutrition support. J Clin Gastroenterol 2004;38:403-407.

-68 Kaufman S, Atkinson JB, Bianchi A, Goulet O, Grant D, Langnas A, et al: Indications for pediatric intestinal transplantation: a position paper of the American Society of Transplantation. Pediatr Transplant 2001:5:80-87.

69 Colomb V, Jobert-Giraud A, Lacaille F, Goulet O, Fournet JC, Ricour C: Role of lipid emulsions in cholestasis associated with long-term parenteral nutrition in children. JPEN J Parenter Enteral Nutr 2000;24:345-350.

70 Iyer K, Horslen S, Torres C, et al: Histology is not predictive of functional liver recovery in parenteral-nutrition associated liver dysfunction (abstract). Pediatr Transplant 2003;7:69.

71 Bueno J, Ohwada S, Kocoshis S, et al: Factors impacting the survival of children with intestinal failure referred for intestinal transplantation. J Pediatr Surg 1999;34:27-33.

72 Fryer J, Pellar S, Ormond D, Koffron A, Abecassis M: Mortality in candidates waiting for combined liver-intestine transplants exceeds that for other candidates waiting for liver transplants. Liver Transplant 2003;9:748753.

73 Grant D, Abu-Elmagd K, Reyes J, Tzakis A, Langnas A, Fishbein T, Goulet O: Farmer D, on the behalf of the Intestine Transplant Registry: 2003 report of the intestine transplant registry: a new era has dawned. Ann Surg 2005; 241:607-613.

74 Beath SV, Booth IW, Murphy MS, et al: Nutritional care and candidates for small bowel transplantation. Arch Dis Child 1995; 73:348_ 350 .

75 Fishbein TM, Schiano T, LeLeiko N, et al: An integrated approach to intestinal failure. Results of a new program with total parenteral nutrition, bowel rehabilitation, and transplantation. J Gastrointest Surg 2002;6:554-562.

76 Guarino A, De Marco G, Italian National Network for Pediatric Intestinal Failure: Natural history of intestinal failure, investigated through a national network-based approach. J Pediatr Gastroenterol Nutr 2003;37:136141.

77 Kocoshis SA, Beath S, Booth IW, Oliva GA, Goulet O, Kaufman SS, Lai HS, Luque C, Ohtsuka Y: Intestinal failure and small bowel transplantation, including clinical nutrition: Working Group report of the second World Congress of Pediatric Gastroenterology, Hepatology and Nutrition. J Pediatr Gastroenterol Nutr 2004;39(suppl 2):S655-S661. 